\title{
Correction to: Remarks About Spatially Structured SI Model Systems with Cross Diffusion
}

Verónica Anaya, Mostafa Bendahmane, Michel Langlais

and Mauricio Sepúlveda

Correction to:

Chapter "Remarks About Spatially Structured SI Model Systems with Cross Diffusion" in: B. N. Chetverushkin et al. (eds.), Contributions to Partial Differential

Equations and Applications, Computational Methods in Applied Sciences 47, https://doi.org/10.1007/978-3-319-78325-3_5

The book was inadvertently published with chapter author's incorrect given name. This information has been updated from "Vanaya Anaya" to "Verónica Anaya" in the initially published version of chapter "Remarks About Spatially Structured SI Model Systems with Cross Diffusion". 